\title{
Universality of Mass to Area Ratio: from Biological to Astronomical Structures C Sivaram
}

Indian Institute of Astrophysics

Bangalore - 560 034, India

\section{Kenath Arun ${ }^{1}$}

Christ Junior College

Bangalore - 560 029, India

\section{Kiren $\mathrm{O} V$}

St. Josephs Indian Composite PU College

Bangalore - 560 001, India

\begin{abstract}
For a hierarchy of large scale structures, like galaxies, galaxy clusters, superclusters, etc. the requirement that their gravitational (binding) self-energy density must at least equal or exceed the background repulsive dark energy density implies a universal mass-radius relation given by $M / R^{2} \approx 1 \mathrm{~g} / \mathrm{cm}^{2}$. Here we point out that this mass-radius relation also holds for important individual biological structures, and even various organisms as a whole such as humans, trees, and to behemoths like whales, even though the physics is different. Here we try to understand this universality from two different physical considerations. We also point out the coincidence that the densities of most biological entities are close to that of water, and that the average density of main-sequence stars and giant planets are close to this density. We give a physical basis for the same.
\end{abstract}

Key Words: Mass/area; biological structures; gravitational self-energy; dark energy

\footnotetext{
${ }^{1}$ Corresponding Author: e-mail: kenath.arun@cjc.christcollege.edu Telephone: +91-80-4012 9292; Fax: +91-80- 40129222 Orcid id: 0000-0002-2183-9425
} 


\section{Introduction}

In recent papers (Sivaram et al., 2013; Sivaram and Arun, 2012; 2013) following earlier works (Sivaram, 1993; 2000) we had highlighted that astronomical structures ranging from super clusters of galaxies, to globular clusters follow more or less a mass to radius squared ratio, i.e. $M / R^{2}$ of the same numerical magnitude given by, $\frac{M}{R^{2}} \approx 1 \mathrm{~g} / \mathrm{cm}^{2}$. Curiously this also holds for the universe as a whole $\left(M \approx 10^{56} \mathrm{~g} ; R \approx 10^{28} \mathrm{~cm}\right)$. We interpreted this in terms of the universality of dark energy dominating the universe and as current observations suggest it could be nothing other than Einstein's cosmological constant $\Lambda \approx 10^{-56} \mathrm{~cm}^{-2}$. We had the universal relation:

$$
\frac{M}{R^{2}}=\sqrt{\Lambda} \frac{c^{2}}{G} \approx 1 \mathrm{~g} / \mathrm{cm}^{2}
$$

(Here $c$ is velocity of light and $G$ the Newton's Gravitational constant.)

It could be understood, as a balance pressure of Dark Energy density $\left(\sim \frac{\Lambda c^{4}}{8 \pi G}\right)$, balanced by the gravitational force pressure energy density $\left(\frac{G M^{2}}{8 \pi R^{4}}\right)$, thus giving the above relation (for the observed $\Lambda \approx 10^{-56} \mathrm{~cm}^{-2}$ ). It was also noted (Sivaram, 1993; 2000) that even the electron and a typical hadron follow the same $M / R^{2}$ value (e.g. for electron $m_{e} \approx 10^{-27} \mathrm{~g} ; r_{e}=10^{-13} \mathrm{~cm}$ again implying $\left.\frac{M}{R^{2}} \approx 1 \mathrm{~g} / \mathrm{cm}^{2}\right)$. Several other examples for other particles are also given in Sivaram (1994). It is remarkable that these typical mass and radius of ancient (primordial) galaxies also seem to satisfy, the $M \propto R^{2}$ relation ( $M, R$ being the mass and radius of the galaxy). This ancient galaxy is found to be relatively small having a size about a tenth of the Milky Way, i.e. it is about one kiloparsec across. Also its mass turns out to be about one per cent of that our galaxy (Laporte et al., 2017).

\section{Mass to Area Ratio in Biological Structures}

We now point out that the $M / R^{2}$ ratio of more or less the same value also holds for biological structures. The body mass index given by $M / \mathrm{L}^{2}$ e.g. $M \approx 50 \mathrm{~kg} ; L=2 \mathrm{~m}$, is typically again $\sim 1 \mathrm{~g} / \mathrm{cm}^{2}$. This also holds for individual important structures in the body like the brain 
and skin. For instance the typical brain mass is $1500 \mathrm{~g}$, and the surface area of the brain (ironed out of the convolutions) is about $2000 \mathrm{~cm}^{2}$. This gives an $\frac{M}{L^{2}} \approx 1 \mathrm{~g} / \mathrm{cm}^{2}$. The total mass of skin is $5 \mathrm{~kg}$ covering one square metre, this gives $0.5 \mathrm{gm} / \mathrm{cm}^{2}$. Same value also holds for other structures, including elephants, trees (a hundred metre tree weighs 200 tons for instance). For a mouse (5g mass and $2 \mathrm{~cm}$ length) again gives an $\frac{M}{L^{2}} \approx 1 \mathrm{~g} / \mathrm{cm}^{2}$. For biological structures it is difficult to find a general physical basis (like we had the balance between repulsive dark energy and gravitational force for large astronomical structures) (Sivaram et al., 2014). Here we provide possible physical explanations for this, based on basic physics. For objects in motion, in an atmosphere (or in a medium) the weight, $M g$, is to balance the drag force of the medium. Thus:

$$
M g=C_{D} \rho_{a t m} A \mathrm{v}^{2}
$$

(This equation was found useful for understanding a wide range of biological characteristics including bird flight, wing speeds, etc.)

This gives (with the drag coefficient, $C_{D} \approx 1$ ):

$$
\frac{M}{A}=\frac{\rho_{a t m} \mathrm{v}^{2}}{\mathrm{~g}}
$$

For a planet like Earth, acceleration due to gravity, $g=10^{3} \mathrm{~cm} / \mathrm{s}^{2}$, the atmospheric density, $\rho_{\text {atm }} \approx 10^{-3} \mathrm{~g} / \mathrm{cc}$, and a typical velocity for various creatures, $\mathrm{v}$ of about $10 \mathrm{~km} / \mathrm{h}$, in equation (3), we have $M / R^{2} \approx 1 \mathrm{~g} / \mathrm{cm}^{2}$, agreeing with equation (1).

It turns out that in the case of underwater behemoths like whales and other cetaceans it could be somewhat different, though to within an order of magnitude. For cetaceans submerged in fluids (e.g. water), we have the drag force, $C_{D} \rho_{W} A \mathrm{v}^{2}\left(A \sim R^{2}\right.$ is the area, $\rho_{W}$ is the density of water, $\mathrm{v}$ is the velocity with which the organism can move on average). Balancing this to the weight $M g$ again gives,

$$
\frac{M}{A} \approx \frac{C_{D} \rho_{W} \mathrm{v}^{2}}{\mathrm{~g}}
$$

If $\mathrm{v}$ is about $20 \mathrm{~km} / \mathrm{h}, g=10^{3} \mathrm{~cm} / \mathrm{s}^{2}$, and in the fluid, $C_{D}=0.1-0.2$, then we have, $\frac{M}{R^{2}} \approx 2.5-5$

It is interesting to conjecture whether cetaceans existing on say, Europa ocean (below the surface), or Titan (their gravity being $1 / 7$ ) have a similar $M / R^{2}$. If we assume $M / R^{2}$ does not vary much, on a lower $g$ planet $\mathrm{v}$ would be higher (since $\mathrm{v}^{2} / g$ is a constant). A lighter fluid 
would give a higher velocity etc. (Sivaram et al., 2016). One can extrapolate the above results to habitable exoplanets with different atmospheric densities and surface gravities. For example a higher $g$ would imply a smaller mass for a given area and a higher atmospheric density implies a higher mass for a given area, which are both physically reasonable conclusions (less mass is suitable for higher $g$, more mass for more resistance to motion). Generally planets with a higher atmospheric density would be expected to have a higher $g$. Therefore, $M / A$ (being proportional to $\rho / g$ ) may not vary too much even for exoplanets which are potentially habitable.

\section{Mass to Area Ratio from Kleiber's law}

In a recent paper (Sivaram et al., 2017), we had invoked Kleiber's law in the context of bioenergetics. Kleiber's law is given by:

$$
B=a M^{3 / 4}
$$

Where $B$ is the basal metabolic rate, $M$ is the mass of the organism, $a \sim 90 \mathrm{kcal}^{\prime} \mathrm{kg}^{3 / 4}$ is a constant. The relation is seen to hold over several orders (in mass) especially among mammals and warm blooded species. Typically a $60 \mathrm{~kg}$ individual has a $B$ about $3000 \mathrm{kcal} / \mathrm{day}$. Hence this is the minimum energy required to generate body heat to keep the temperature to about $37^{\circ} \mathrm{C}$ $(\sim 310 \mathrm{~K})$. At this temperature the emission is in the infrared (around 7-10 microns). As individuals, we glow at about $120 \mathrm{~W}$ in the infrared. The radiation power emitted at $310 \mathrm{~K}$, for a body area of $1 \mathrm{~m}^{2}$, is given by Stefan's law, $P=\sigma A T^{4} \approx 100 \mathrm{~W} \approx 10^{9} \mathrm{erg} / \mathrm{s}$, which corresponds to the value above.

The heat stored is, $H=M S T$, the specific heat, $S \sim 1$, since we are mostly water, $T \sim$ $30^{\circ} \mathrm{C}, M \approx 7 \times 10^{4} \mathrm{~g}$, this gives, $H \sim 2200 \mathrm{kcal}$, which is the $B$ for a typical individual of mass $60 \mathrm{~kg}$. For a 2.5 ton elephant, $B \sim 70000 \mathrm{kcal} / \mathrm{day}$. So $100 \mathrm{~W}$ power emitted (in the IR) corresponds to a daily heat output of $\sim 2500 \mathrm{kcal}$.

As can be seen, all the above relations are nicely consistent. To understand $M / R^{2}$ from Kleiber's law, we equate $B$ to heat generated (as a minimal requirement), $B \approx P \times$ number of seconds in a day:

$$
B=a M^{3 / 4} \approx \sigma A T^{4} \times 10^{5}
$$

This gives, $\frac{M}{A} \approx 1 \mathrm{~g} / \mathrm{cm}^{2}$ (Sivaram et al., 2017). 
As Kleiber's law is universal, so also would be the $M / R^{2} \approx 1 \mathrm{~g} / \mathrm{cm}^{2}$. Thus two different arguments based on different physical considerations both seem to imply the same value for $M / R^{2}$ for a wide class of bioorganisms.

\section{Densities of Biological Structures}

There is another curious coincidence in that the densities of most biological entities are close to that of water. This is understandable as most biological structures are dominantly composed of water; we are seventy per cent water by weight, blood plasma is ninety per cent water, as also many vegetables, fruits, etc. What is curious is that the average density of mainsequence stars like the Sun and Sirius, as also giant planets like Jupiter etc. are again close to water density.

Even terrestrial planets have densities a few times that of water. Here the explanation (i.e. for stellar bodies) is quite straightforward. The average distance between atoms is the Bohr radius, i.e. $10^{-8} \mathrm{cms}$. So in a volume of $10^{-24} \mathrm{~cm}^{3}$, we have a mass of $10^{-24} \mathrm{gm}$ (i.e. the proton mass since the electron mass is negligible). This gives $1 \mathrm{~g} / \mathrm{cc}$, i.e. water density. The radius of the sun is $N^{1 / 3} \mathrm{x}$ Bohr radius, $N$ being the total number of atoms (mainly hydrogen) in the Sun. $N=10^{57}$ for the Sun. So this gives the solar radius which is just what is observed. $N^{1 / 3}$ just implies that the average separation is the Bohr radius. For heavier elements, constituting planets (rocky material) the density would be a few times higher. If we take total number of atoms, say $10^{28}$ in a person, then this implies a linear dimension of $\sim 10^{2} \mathrm{~cm}$, which is the observed value. (Sivaram et al., 2014)

When a star evolves, the cores get compressed, the atoms being squeezed much closer, so the densities now become much higher. In a neutron star, the separation between neutrons is the nuclear radius of a fermi, so for $N=10^{57}$ (a solar mass) we get a radius of ten kilometres, which is the neutron star radius. The density is a hundred trillion times that of water. For white dwarfs the separation between squeezed 'atoms' is the electron Compton length and this multiplied by $N^{1 / 3}$ gives the white dwarf radius, similar to that of the Earth. The density now is a million times that of water.

\section{Reference:}

N. Laporte et al., Astrophys. J. Lett. 837, L21 (2017)

C. Sivaram, K. Arun, Open Astron. J. 5, 7 (2012)

C. Sivaram, K. Arun, Astrophys. Space Sci. 348, 217 (2013) 
C. Sivaram, K. Arun, O.V. Kiren, Open Astron. J. 6, 90 (2013)

C. Sivaram, K. Arun, O.V. Kiren, Astrobiology Newsletter 7(3), 3 (2014)

C. Sivaram, K. Arun, O.V. Kiren, Astrobiology Newsletter 9(3), 7 (2016)

C. Sivaram, K. Arun, O.V. Kiren, Int. J. Astrobiol. (2017) https://doi.org/10.1017/ S1473550417000386

C. Sivaram, Astrophys. Space Sci. 207, 317 (1993)

C. Sivaram, Astrophys. Space Sci. 215, 185 (1994)

C. Sivaram, Astrophys. Space Sci. 271, 321 (2000) 